\title{
DESAFIOS E OPORTUNIDADES DO USO DA TECNOLOGIA NA PRÁTICA DOCENTE: UMA REVISÃO EM TORNO DO TPACK NO BRASIL
}

\section{Challenges and Opportunities for the Use of Technology in Teaching Practices: a Review on TPACK in Brazil}

\author{
Maykon Nise Furtado ${ }^{1}$ \\ Guilherme Laranjeira Mendonça Oliveira \\ Ismael Moura Parede 3 \\ Carlos Alexandre Felício Brito ${ }^{4}$
}

\begin{abstract}
Resumo: A utilização da tecnologia no ambiente educacional faz-se cada vez mais presente, de acordo com as demandas sociais eminentes, e, com isso, sujeita que os professores saibam como lidar com tais recursos, potencializando suas práticas pedagógicas, estabelecendo assim a importância do modelo TPACK - Technological Pedagogical And Content Knowledge (Conhecimento Tecnológico Pedagógico do Conteúdo). Esta pesquisa, de caráter qualitativo, objetiva revisar, de forma sistemática, a literatura existente, no espaço temporal entre 2015 e 2020, que verse sobre o desenvolvimento do modelo TPACK nas pesquisas nacionais. Objetiva-se verificar as perspectivas e identificar os desafios dessa proposta pedagógica. Diante disso, os resultados apresentam a análise de 20 produções acadêmicas, tendo o ápice da quantidade de publicações em 2017, sendo que metade dos estudos analisados são provenientes da região sul e a maioria são artigos publicados em revistas. Outro aspecto importante é a precariedade de estudos empíricos, sendo mais da metade revisões da literatura.
\end{abstract}

Palavras-chave: TPACK. Conhecimento Tecnológico Pedagógico do Conteúdo. TDIC.

Abstract: The use of technology in the educational environment is becoming more and more present, according to the eminent social demands, and with this, it makes it possible for teachers to know how to deal with such resources, enhancing their pedagogical practices, thus

\footnotetext{
${ }^{1}$ Mestrando em Educação pela Universidade Municipal de São Caetano do Sul (USCS), Especialista em Gestão de Pessoas pela UNINOVE (2013), Professor do Serviço Nacional de Aprendizagem Comercial (SENAC-SP). Orcid: https://orcid.org/0000-0001-7673-2417. E-mail: ismael.parede@prof.uscs.edu.br.

2 Mestrando em Educação pela Universidade Municipal de São Caetano do Sul (USCS), Licenciado em Matemática pela UNIMES (2020), Professor da rede estadual de São Paulo. Orcid: https://orcid.org/0000-0001-5707-1000. E-mail: laranjeiraguilherme@gmail.com.

3 Mestrando em Educação pela Universidade Municipal de São Caetano do Sul (USCS), Especialista em Engenharia de Redes e Telecomunicações pela Universidade São Judas Tadeu (2003), Professor de Graduação da Universidade Municipal de São Caetano do Sul (USCS) e da Faculdade de Tecnologia do Estado de São Paulo (FATEC). Orcid: https://orcid.org/0000-0002-4188-6595. E-mail: ismael.parede@prof.uscs.edu.br.

${ }^{4}$ Doutor em Educação Física pela Universidade Estadual de Campinas (2005). Professor do Programa de Mestrado Profissional em Educação (PPGE) na Universidade Municipal de São Caetano do Sul. Orcid: https://orcid.org/0000-0002-0060-8644. E-mail: carlos.brito@prof.uscs.edu.br.
} 
establishing the importance of the model TPACK. This qualitative research aims to systematically review the existing literature, in the period between 2015 and 2020, which deals with the development of the TPACK model in national surveys, aims to verify the perspectives and identify the challenges of this

pedagogical proposal. Therefore, the results show the analysis of 20 academic productions, with the peak of the number of publications in 2017, with half of the studies analyzed coming from the southern region and the majority being articles published in magazines. Another important aspect is the precariousness of empirical studies, more than half of which are literature reviews.

Keywords: TPACK. Technological Pedagogical And Content Knowledge. TDIC.

\section{Introdução}

O uso da tecnologia no âmbito educacional torna-se cada vez mais importante, de acordo com os novos avanços da sociedade, modificando o paradigma tradicionalista da sala de aula (MORAN, 2001). Nesse sentido, há necessidade de repensarmos como os professores estão sendo preparados para atuar em sala de aula com auxílio da tecnologia.

Assim, com o desenvolvimento das demandas sociais, e de como elas são influenciadas pelo conhecimento formal, emerge o conceito de web 2.0, o qual vem se modificando desde o início do século XXI, quando o uso da internet para comunicação e informação se massificou por meio das novas ferramentas digitais e do conceito de partilha, bem como com o uso das redes sociais, caracterizando assim, a denominação de "Web Social" (GIL, 2014).

No entanto, não bastou apenas a disponibilização da informação no meio digital, tornou-se necessário organizá-la, de forma sistematizada, a fim de contextualizar o conhecimento, caracterizando assim, a passagem da sociedade da informação para a sociedade da comunicação. Diante disso, o ambiente de partilha passa a ter a possibilidade de ocorrer de forma síncrona, com amparo das novas ferramentas digitais, caracterizando o conceito de web 3.0 .

Busca-se, no processo de ensino e aprendizagem, desenvolver de forma massiva procedimentos para que os docentes possam utilizar essa variedade de tecnologias da informação e comunicação, as quais podem ser úteis no ambiente educacional. Contudo, apropriar-se dessas ferramentas, fazendo com que elas de fato colaborem com o ensino e a produção de conhecimento em sala de aula, não é tarefa fácil, pois exige uma formação contínua dos docentes.

O presente estudo torna-se de relevância social por abordar um assunto contemporâneo, uma vez que outras pesquisas já foram realizadas em âmbito internacional e nacional, até o período de 2015 (NOGUEIRA; PESSOA; GALLEGO, 2015; MANEIRA; GOMES, 2016). Assim, este artigo objetiva compreender como o Conhecimento Tecnológico Pedagógico do Conteúdo - Technological Pedagogical Content Knowledge (TPACK) (MISHRA; KOEHLER, 2006), está sendo pesquisado na literatura nacional, por meio de uma revisão sistematizada da literatura em uma escala temporal de 2015 a 2020. 


\section{Constituição do TPACK}

O avanço tecnológico trouxe profundas transformações na educação, fazendo com que os professores se adaptem às demandas sociais. Assim, esse progresso proporcionou uma nova dimensão no saber-fazer deste profissional, condizente ao conhecimento tecnológico, o qual torna-se relevante aos professores devido ao desenvolvimento tecnológico na educação.

Num primeiro momento, antecedente à abordagem tecnológica, a principal discussão envolvia uma estrutura conceitual para a tecnologia educacional, com base na formulação que Shulman descrevia como o "conhecimento do conteúdo pedagógico". Todavia, limitando-se às demandas sociais da época, em que a tecnologia não era tão avançada, mas estava em iminência à progressão. Nesse sentido, Shulman (1986) denominava de Conhecimento Pedagógico de Conteúdo (CPC), afirmando que esse conhecimento inclui "tópicos regularmente ensinados de uma área específica de conhecimento, as representações mais úteis de tais ideias, as analogias mais poderosas, ilustrações, exemplos, explanações e demonstrações" (SHULMAN, 1986, p. 9).

$\mathrm{Na}$ Figura 1, a seguir, é apresentado a intersecção entre o conhecimento do conteúdo e o conhecimento pedagógico:

Figura 1 - Conhecimento Pedagógico de Conteúdo

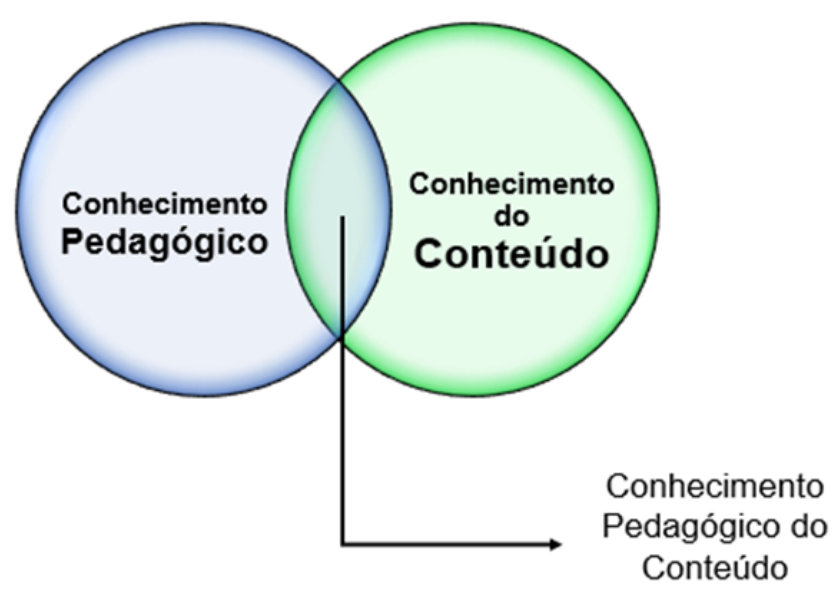

Fonte: Adaptado de Shulman (1986)

Entretanto, diante da influência das tecnologias digitais na sociedade (MISHRA; KOEHLER, 2006), percebeu-se a necessidade do desenvolvimento do Conhecimento Tecnológico na formação do professor, ou seja, da habilidade que consiste em dominar as Tecnologias Digitais de Informação e Comunicação (TDIC). Além disso, os autores afirmam que o Conhecimento Tecnológico deve relacionar-se com os conhecimentos de Lee Shulman, o CPC, assim, estabeleceu-se o Conhecimento Tecnológico Pedagógico do Conteúdo Technological Pedagogical Content Knowledge - TPACK (KOEHLER; MISHRA, 2006).

Esse modelo surgiu em 2006, o qual é resultado de um programa de pesquisa cujo foco é o desenvolvimento profissional de docentes. O TPACK serve como fonte de pesquisa de muitos autores que investigam sobre a formação de professores.

Desse modo, a revisão sistemática deste artigo tem como referencial teórico o modelo TPACK, que é composto pela interseção de três elementos: conhecimento de conteúdo (CK - 
content knowledge); conhecimento pedagógico ( $\mathrm{PK}$ - pedagogical knowledge) $\mathrm{e}$ conhecimento tecnológico ( $\mathrm{TK}$ - technological knowledge), conforme Figura 2:

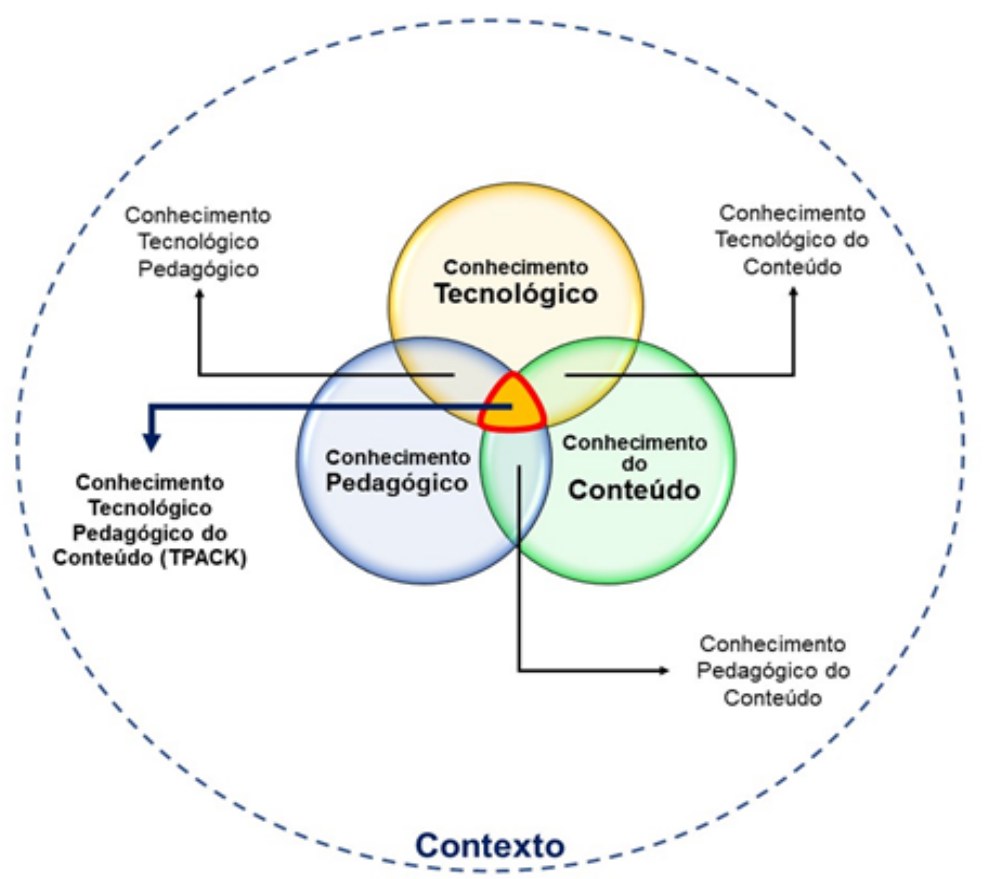

Fonte: Adaptado de Koehler e Mishra (2006, p. 1025)

Na Figura 2, observa-se quatro interseções gerando novos conhecimentos, que são: (1) Conhecimento Pedagógico de Conteúdo, (2) Conhecimento Tecnológico de Conteúdo, (3) Conhecimento Tecnológico Pedagógico, (4) Conhecimento Tecnológico Pedagógico de Conteúdo (TPACK). Sendo assim, no quadro 1 são apresentadas as definições de cada uma dessas interseções:

Quadro 1 - Síntese das interseções dos elementos do TPACK

\begin{tabular}{|c|l|}
\hline $\begin{array}{c}\text { Conhecimento Pedagógico do } \\
\text { Conteúdo (PCK) }\end{array}$ & $\begin{array}{l}\text { É a competência de ensinar com arte e ciência, é dominar o } \\
\text { conteúdo e saber como conduzi-lo. }\end{array}$ \\
\hline $\begin{array}{c}\text { Conhecimento Tecnológico de } \\
\text { Conteúdo (TCK) }\end{array}$ & $\begin{array}{l}\text { É a competência, do professor, para avaliar a melhor } \\
\text { tecnologia que se relaciona com o conteúdo. }\end{array}$ \\
\hline $\begin{array}{c}\text { Conhecimento Tecnológico } \\
\text { Pedagógico (TPK) }\end{array}$ & $\begin{array}{l}\text { É a competência, do professor, para avaliar a TDIC mais } \\
\text { adequada para a estratégia de ensino aprendizagem } \\
\text { pretendida. }\end{array}$ \\
\hline $\begin{array}{c}\text { Conhecimento Pedagógico } \\
\text { Tecnológico de Conteúdo (TPACK) }\end{array}$ & $\begin{array}{l}\text { É a competência para dominar métodos pedagógicos de } \\
\text { ensino que utilizam tecnologias digitais de maneiras } \\
\text { construtivas para desenvolvimento de um conteúdo. }\end{array}$ \\
\hline
\end{tabular}

Fonte: Adaptado de Andrade, Alencar e Coutinho (2019) 
Portanto, o Conhecimento Tecnológico e Pedagógico do Conteúdo, conhecido internacionalmente como TPACK (Technological Pedagogical Content Knowledge) são técnicas pedagógicas que usam tecnologias de forma construtiva para ensinar conteúdos (ANDRADE; ALENCAR; COUTINHO, 2019).

\section{Percurso Metodológico}

Nesta pesquisa, de abordagem qualitativa, adotou-se a revisão sistemática da literatura, a qual busca identificar, analisar e sintetizar as publicações que versam sobre a temática do TPACK, relacionando os conceitos desse modelo, desde sua origem até a atualidade (LEITE, 2018). Neste estudo de caráter acadêmico, revisamos artigos, anais, teses, dissertações e monografias, refletindo sobre as contribuições e avanços do TPACK para a sociedade.

Para sistematizar a pesquisa, foram acatadas algumas perguntas norteadoras, a saber: a) quais tipos de pesquisa e publicações na área da educação, que versam sobre o modelo TPACK, estão presentes na literatura no Brasil? b) quais os principais enfoques e temáticas desses estudos? c) que contribuições e avanços do TPACK, essas pesquisas proporcionam para a sociedade?

Assim, o estudo foi realizado no período de setembro e outubro de 2020, utilizando as plataformas: Google Académico, Scientific Electronic Library Online (SciELO) e Biblioteca Digital Brasileira de Teses e Dissertações (BDTD), entrelaçando os resultados de acordo com a combinação dos descritores utilizados: "TPACK"; "TPCK" e "Conhecimento Tecnológico Pedagógico de Conteúdo". Entretanto, considerou-se apenas os estudos no contexto lusófono, língua portuguesa, publicados em âmbito nacional, e no espaço temporal entre 2015 e o primeiro semestre de 2020.

No âmbito deste estudo, os pesquisadores localizaram 203 entradas, na primeira fase do processo, para identificar as publicações recorrentes. Após a análise individual de cada resumo, foi possível selecionar apenas 20 produções acadêmicas, que contemplam os critérios de seleção estabelecidos, excluindo aquelas que não versam sobre a temática do modelo TPACK e as que podem ser consideradas publicações repetidas dentre as identificadas.

Os resultados apurados nesta pesquisa dizem respeito apenas ao contexto lusófono e às publicações brasileiras. Sendo assim, destacam-se artigos em periódicos e anais de congressos, dissertações, teses e monografias. Logo, aqui apresentamos os avanços nos estudos que envolvem o modelo TPACK, no espaço temporal estabelecido e com os indicadores norteadores do estudo, a saber: ano de publicação, tipo de publicação, tipo de estudo, classificação da instituição na qual o autor está vinculado, área do conhecimento e etapa de ensino.

\section{Resultados}

Conforme os procedimentos sistemáticos de revisão de literatura utilizados neste estudo, obteve-se os resultados logo apresentados em detalhe, baseados nos indicadores supracitados. 
Gráfico 1 - Número absoluto de publicações da temática pesquisada (TPACK) em função dos anos

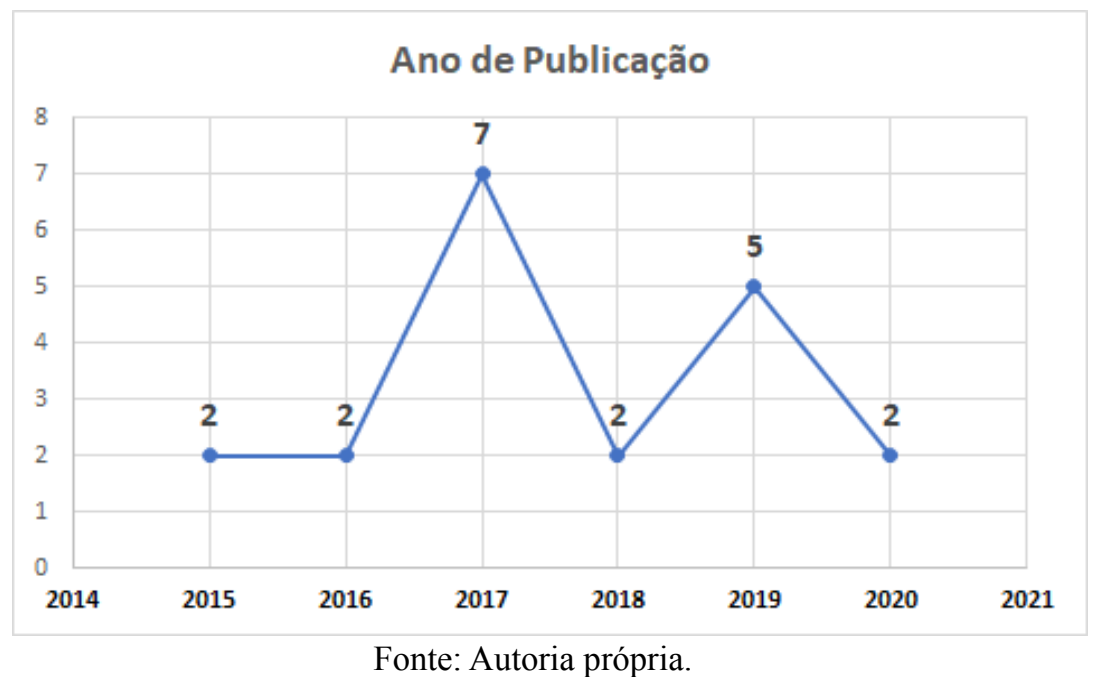

A partir da análise do gráfico 1, podemos observar que, diante do espaço temporal adotado para este estudo, a quantidade de publicações teve seu auge nos anos de 2017 e 2019, contemplando sete e cinco publicações, respectivamente, diferente dos demais anos.

Em relação ao tipo de publicação, diante das pesquisas em âmbito nacional, foi possível identificar uma frequência maior em publicações de artigos em revistas $(n=7)$, similar às publicações de dissertações de mestrado $(n=6)$, quando comparada aos artigos publicados em anais $(n=4)$, teses de doutorado $(n=2)$ e monografia $(n=1)$.

Quanto à distribuição das publicações, de acordo com as regiões das instituições vinculadas aos autores, podemos identificar que 50\% destas encontram-se na região Sul $(\mathrm{n}=10)$ do Brasil, contemplando apenas $30 \%$ das instituições localizadas na região Sudeste $(n=6)$ e $20 \%$ das instituições na região Norte $(n=4)$. Não obstante, foi observado que $90 \%$ $(\mathrm{n}=18)$ dessas instituições pertencem ao domínio público e apenas $10 \%(\mathrm{n}=2)$ das instituições são privadas. Sendo que, dentre estas, dez instituições são da esfera Estadual (55,5\%) e oito instituições são da esfera Federal (44,4\%).

No que diz respeito ao tipo de estudo, pode-se observar uma divisão equitativa entre pesquisas qualitativas empíricas $(n=9)$ e qualitativas de revisão/ensaio $(n=10)$. Embora ambas sejam consideradas qualitativas, é relevante considerar que a pesquisa empírica analisa as respostas, ou observa situações e participantes, em contraste com a pesquisa teórica que consiste na revisão da literatura e ensaios, os quais têm o foco na teoria científica, sem necessidade de envolver outras pessoas. Vale ressaltar, a existência de uma pesquisa quantitativa sobre o TPACK $(\mathrm{n}=1)$. 
Gráfico 2 - Frequência do tipo de pesquisa qualitativa e quantitativa

Tipo de Pesquisa

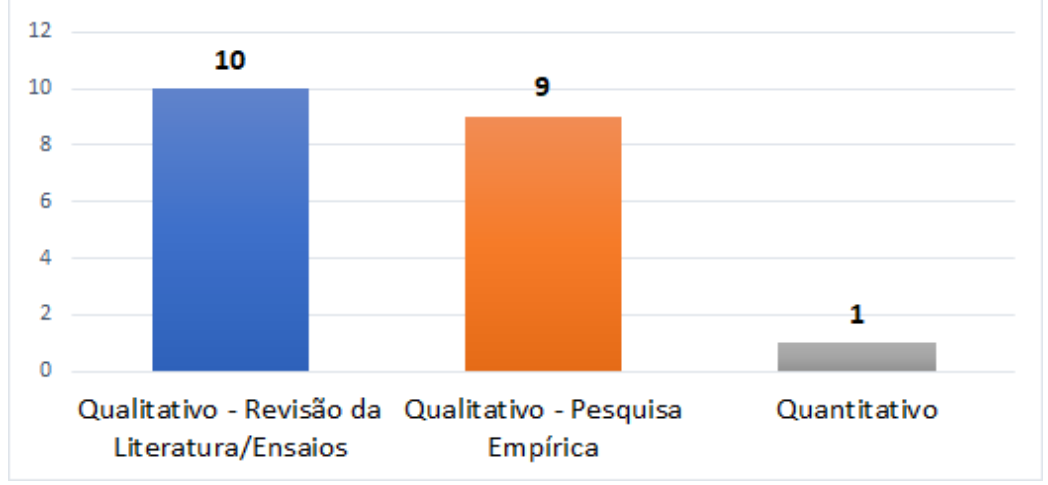

Fonte: Autoria própria.

Em relação às etapas de ensino, evidenciadas nos estudos em análise, a maioria das produções acadêmicas tem como objeto de estudo a educação básica $(n=10)$, sendo dois estudos no âmbito dos anos iniciais e oito estudos com foco nos anos finais. Com base nos anos finais da educação básica, é possível constatar uma semelhança na quantidade de estudos científicos $(\mathrm{n}=2)$ nas disciplinas de Química, Geografia e Ciências. O mesmo acontece nas disciplinas de Matemática e Biologia, com apenas uma produção que versa sobre cada tema. Ainda foi possível observar que as produções que versam sobre o ensino superior, e as sem especificações de etapa de ensino, conduzem a quatro publicações, respectivamente.

Gráfico 3 - Frequência nas pesquisas quanto ao seu enfoque

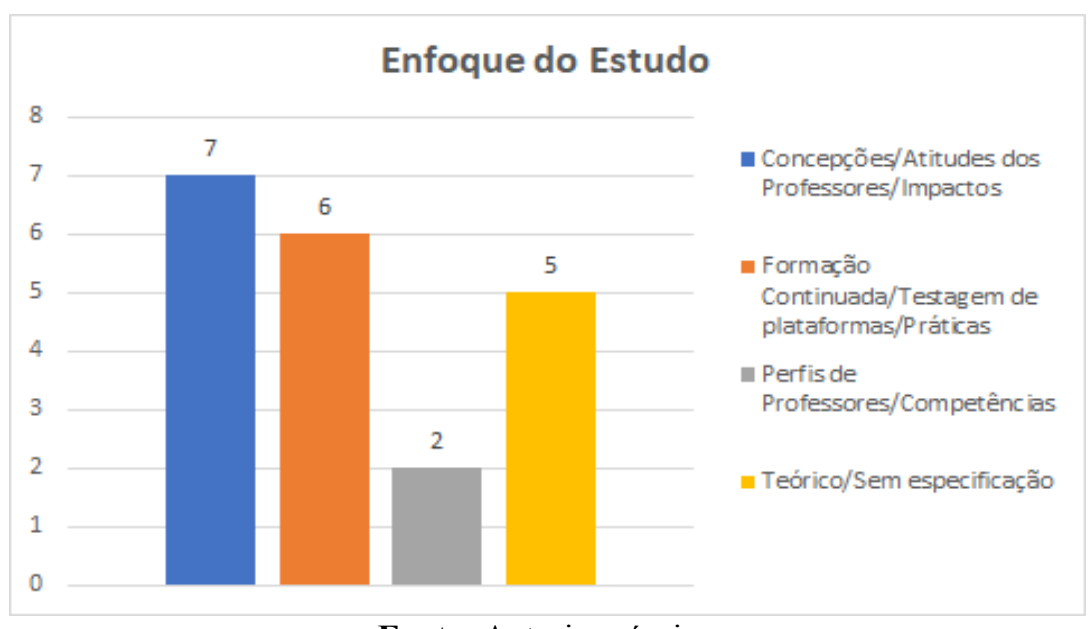

Fonte: Autoria própria.

A partir da análise do conteúdo disposto nas produções, foi possível segmentá-las em quatro descritores. Portanto, pode-se notar que existe uma diferença gradativa, uma unidade entre os descritores, com exceção dos perfis de professores e competências $(n=2)$. No entanto, podemos perceber que o auge da quantidade de publicações $(n=7)$ direcionam as concepções, as atitudes dos professores e os impactos; inobstante a formação continuada, a testagem de plataformas e práticas, com uma pequena diferença $(n=6)$, e, por sequência, as produções apenas com aporte teórico e sem especificações $(n=5)$. 


\section{Discussão dos resultados}

Desde o início da proposta do modelo teórico de Mishra e Koehler (2006), diversas pesquisas foram desenvolvidas para compreender melhor os conceitos que envolvem TPACK, e a partir da análise do conteúdo das publicações selecionadas, foi possível verificar que as produções realizadas no espaço temporal adotado não possuem conexões entre si, ou seja, apesar das pesquisas utilizarem Mishra e Koehler como referencial teórico, não apresentam avanços científicos a partir das contribuições dos pesquisadores atuais, com intuito de agregar na evolução dos conceitos envolvidos no framework TPACK.

Em 2015, houve duas publicações. A primeira pesquisa trata de uma revisão sistemática, elaborada por Rolando, Luz e Salvador (2015), que ressaltam que a maior parte das pesquisas realizadas entre 2006 e 2014 não estão relacionadas à formação de professores, mas, sim, à compreensão de como as tecnologias são utilizadas no processo de ensino-aprendizagem, bem como comentam que a quantidade de publicações vem crescendo. Além disso, destacam, nas considerações finais, a "necessidade de se buscar caminhos metodológicos que sejam eficazes em aferir adequadamente os resultados da aplicação de determinadas teorias na prática educacional" (ROLANDO; LUIZ; SALVADOR, 2015, p. 183); tal afirmação é importante, visto que um método de referência para medir o nível de TPACK do professor, contribuirá para a formação continuada de professores.

Em relação à segunda publicação, em 2015, trata-se de uma tese de doutorado do Rocha (2015), a qual teve como objetivo explorar as noções dos professores de Geografia sobre o uso do TPACK e o entrelaçamento com sua prática docente. Os resultados mostraram que a relação dos professores com as TDIC acontece de forma limitada, seja por falta de recursos estruturais e instrumentais nas escolas, seja por ausência de formação inicial e continuada. $\mathrm{O}$ autor comenta, em suas considerações finais, que as "técnicas e experiências pedagógicas acumuladas pelos professores durante sua carreira docente devem ser estimuladas a se ajustar aos conteúdos de acordo com a tecnologia ensinada no curso" (ROCHA, 2015, p. 219).

Seguindo a ordem cronológica, no ano de 2016, apresentam-se mais duas publicações, sendo uma o ensaio teórico de Santos e Egido (2016) e a outra, uma dissertação de mestrado de Lang (2016). O ensaio de Santos e Egido (2016) teve como objetivo descrever a origem, o significado e a caracterização do TPACK, com base na teoria de Koehler e Mishra, e inclusive nas conclusões pontuam que, para a utilização deste modelo, seria necessário que o professor tivesse compreensão das técnicas pedagógicas, como uma ferramenta de apoio ao professor.

O objetivo da pesquisa de Lang (2016) foi compreender o desenvolvimento do TPACK de professores do ensino fundamental, participantes de um grupo de formação continuada sobre o uso das TIC. Nas considerações finais, o autor alega que os alunos não possuem vontade para realizar as atividades propostas e que não há envolvimento e interesse. Outro fator importante foi o contexto escolar, sendo que os participantes dos encontros não conseguiam se desligar das circunstâncias de sua escola para possibilitar novos aprendizados, por mais que a escola fosse equipada com recursos tecnológicos, pois era necessário o aprendizado para lidar com eles. Os autores ressaltam a importância da diferenciação entre meio e processo, no que se refere ao uso das TIC, fazendo com que as docentes que compreendiam essa diferenciação, conseguissem desenvolver roteiros na perspectiva do TPACK, e as demais, não.

Rocha e Salvi (2016) discutem sobre a ausência do uso das TDIC no ambiente educacional, logo, os autores consideram a importância da formação continuada para adaptar-se às demandas sociais, uma vez que a formação inicial possibilita. Nesse contexto, 
salientam a relevância do estágio curricular supervisionado na formação inicial e da apropriação das TDIC neste momento.

Observa-se que, no ano de 2017, houve um crescimento de cinco publicações, comparado aos anos de 2015 e 2016, sendo cinco artigos científicos, uma dissertação de mestrado e uma tese de doutorado, totalizando 7 publicações, conforme gráfico 1. Diante disso, pode-se constatar que, neste mesmo ano, ocorreram dois eventos de grande porte, o que contribuiu para o desenvolvimento de novos estudos, a saber: "XI Encontro Nacional de Pesquisa em Educação em Ciências - XI ENPEC" e "IV Seminário Internacional de Representações Sociais, Subjetividade e Educação".

No artigo da Cibotto e Oliveira (2017), a crítica refere-se aos estudos anteriores no contexto lusófono, devido a não contemplarem a integralidade do conceito TPACK. Ainda relataram, em sua pesquisa, uma possível interpretação equivocada dos demais autores, quando utilizada na perspectiva do TPACK. E, ainda, apresentaram algumas traduções diferentes, ocasionando numa possível interpretação equivocada do que os pioneiros nesse contexto proporcionaram.

$\mathrm{Na}$ pesquisa de Sousa, Terçariol e Gitahy (2017), concluiu-se que o uso do modelo TPACK, para abordar os conteúdos curriculares, no contexto de uma licenciatura em Química, poderia contribuir para desenvolver a autonomia e o protagonismo do aluno, diminuindo, assim, a ênfase do professor tradicional que só domina o conteúdo técnico da disciplina.

A tese de doutorado de Rolando (2017) proporcionou uma visão específica sobre as competências do professor de Biologia diante do modelo TPACK. O autor afirma que a utilização do modelo é um referencial teórico testável empiricamente, logo, a disponibilização do instrumento de coleta e da metodologia aplicada, bem como os dados obtidos, podem vir a fornecer subsídios para futuras pesquisas.

Inobstante ao assunto tratado, Santos Neto et al. (2017) realizou uma revisão da literatura acerca da relação entre TPACK e a formação de professores de Ciências da Natureza. Assim, o autor constatou a falta de estudos relacionados à disciplina de Física, como relatado por Nogueira (2015), e sugeriu futuras pesquisas. Diante disso, o autor considera que os resultados da revisão apresentaram estudos genéricos e aplicáveis em diversas áreas do conhecimento; cabe desenvolver estudos específicos e empíricos nas determinadas disciplinas de Ciências da Natureza.

Ainda no mesmo ano, Araújo e Hetkowski (2017) realizaram um artigo com a finalidade de discutir sobre o percurso da sua pesquisa de doutoramento, que versa sobre o desenvolvimento da Educação Científica sobre a temática do TPACK, na etapa da educação básica, não cabendo a possível totalidade conclusiva. Entretanto, a dissertação de Oliveira (2017) apresenta um levantamento bibliográfico em torno do TPACK em três idiomas (Inglês, Português e Espanhol), assim, debruçou-se em afirmar que o modelo do TPACK influenciou no desenvolvimento de pesquisas, em teorias e na formação de professores, nos últimos 12 anos. Logo, Oliveira (2017) conclui sua dissertação sugerindo novas pesquisas que versem sobre o modelo TPACK, ampliando as possibilidades de referenciais teóricos e, ainda, evidenciando-se a compreensão de como utilizar tal conceito nas formações inicial e continuada.

Em relação a 2018, foram identificadas duas produções acadêmicas. Wentz (2018) realizou uma pesquisa com alunos do terceiro ano do Ensino Médio, norteada por um planejamento que buscava entrelaçar as competências do TPACK e a Taxonomia de Bloom, para verificar quais das tecnologias eram mais utilizadas no processo de ensino e aprendizagem da disciplina de Química. O estudo foi conduzido em duas etapas: na primeira delas, foi realizada a elaboração do planejamento e aplicação deste, utilizando os princípios 
em análise. Com isso, sua dissertação possibilitou uma visão colaborativa da utilização das TDIC nos processos de ensino.

O projeto de investigação proposto por Espírito Santo, Cardoso e Santos (2018), teve o intuito de mapear estatisticamente a utilização do modelo TPACK nas práticas de ensino dos professores universitários da rede pública. Com isso, constatou a ausência de infraestrutura, nas instituições de ensino superior, para a integração da tecnologia nas práticas docentes.

A pesquisa de Santos (2019), a partir da análise de um livro didático de Matemática, possibilitou perceber como a tecnologia pode potencializar o aprendizado desta disciplina, além de auxiliar na formação continuada de professores da educação básica.

Melo (2019) apresentou uma revisão de literatura sobre o estado da arte e metodologias para o ensino e aprendizagem dos conceitos estatísticos com auxílio dos smartphones. O autor observa a necessidade de novas práticas e de tecnologias no campo da Matemática, visando a integração dos smartphones nas aulas de Estatística.

Tridico (2019) realizou uma revisão da literatura sobre a formação de professores que utilizam tecnologias e das alterações enfrentadas diante da homologação da Base Nacional Comum Curricular (BNCC). A pesquisa analisa em que medida um curso de formação continuada para professores dos anos iniciais contribui para o desenvolvimento do conhecimento tecnológico, pedagógico e de conteúdo algébrico.

Espíndola e Gianella (2019) apresentaram um trabalho teórico, tendo como objetivo revelar os componentes do modelo do TPACK, relacionando-os com o contexto do Ensino de Ciências, de maneira a contribuir para a pesquisa sobre a integração de TDIC. Esse trabalho foi realizado com participações contributivas de autores do ensino de Ciência e da área da Tecnologia Educacional, com interesse em debater as visões de tecnologia, as potencialidades pedagógicas das TDIC e sua utilização no ensino das Ciências. As autoras apontam para a necessidade de aprofundar as pesquisas, visto que os componentes do TPACK se referem a um campo de conhecimento inatingível em sua totalidade, não obstante a todas as considerações, compreendem a contribuição fornecida pelo modelo TPACK e dos produtos feitos a partir dele e que já são utilizados na tecnologia educativa dentro das especificidades do ensino nas áreas de conteúdo.

Pinheiro, Benvenutti e Favretto (2020) discorreram sobre a estrutura TPACK buscando entender o seu relacionamento com o método de ensino e aprendizagem no sistema utilizado em sala de aula. Buscam entender como o aluno aprende de um jeito saudável e prazeroso, de forma expressiva e inovadora. Fundamentam-se em Koehler e Mishra, que divulgam a obrigação dos professores, de entender pedagogicamente a inserção das tecnologias na sala de aula. Na Educação Básica, a tecnologia digital apresenta-se como probabilidade de modificar os métodos de ensino e aprendizagem, falar a língua dos alunos, acercar-se da realidade digital, receber atenção e apropriar-se do processo de aprendizagem, potencializando, assim, a edificação do conhecimento.

Durante a pandemia, Espíndola, Reses e Ramos (2020) escreveram um artigo que discorre sobre a educação à distância ter sido apontada como solução para os obstáculos do ensino superior, versando também sobre as necessidades de um forte método de formação docente e, então, apresentando o estudo. O estudo estimulou a reconsideração sobre o papel do professor e o direcionou para um ensino mais situado em atividades. A pesquisa debateu que a integração de TDIC não trata de um recurso tecnológico, mas consolida-se em um método de articulação das potencialidades, das ferramentas, dos conhecimentos e dos saberes dos docentes no desenvolvimento de alternativas educativas ao seu ensino.

\section{Considerações Finais}


O conhecimento pedagógico do conteúdo vem se desenvolvendo conforme o tempo, a partir de pesquisas que aplicam e investigam seus conceitos. No entanto, a implantação da tecnologia fez-se de suma importância, uma vez que é um conceito contemporâneo e altamente necessário no desenvolvimento de pesquisas que englobam os três conceitos juntos no âmbito escolar.

Assim, as tecnologias digitais tornaram-se aliadas da educação, exigindo do professor aprender usar tais ferramentas para ensino, de modo que o TPACK contempla essa necessidade e propõe que os saberes docentes evoluam, uma vez que não basta ao professor saber transpor um conteúdo de forma pedagógica, mas, sim, dominar o uso de TDIC nesse processo, com intuito de tornar a aprendizagem significativa e atraente do ponto de vista do aluno.

A presente pesquisa objetivou compreender como o Conhecimento Tecnológico Pedagógico de Conteúdo (TPACK) está sendo pesquisado na literatura nacional. De forma geral, as pesquisas analisadas no espaço temporal de 2015 a 2020 apresentaram conceituação genérica do modelo TPACK, não houve aprofundamento e aplicação prática do modelo. O ano de 2017 foi marcado pelo ápice da quantidade de publicações acadêmicas, tendo 7 produções publicadas. Em relação ao enfoque do estudo, pode-se notar que concepções, atitudes dos professores e impactos foi a categoria com maior número de publicações, contemplando 7 produções acadêmicas.

A maioria dos estudos tratam de procedimentos qualitativos, tendo apenas uma produção que versa sobre a metodologia quantitativa. No entanto, dentre os estudos qualitativos, a maioria são revisões da literatura, o que demonstra que tiveram poucas pesquisas de campo e aplicações do modelo TPACK. Dentre as disciplinas e áreas do conhecimento pesquisadas, não foi possível constatar pesquisas que versassem sobre a disciplina de Física, como apresentado no estudo de Nogueira (2015).

No decorrer do desenvolvimento da pesquisa, foi possível perceber a ausência de estudos correlatos nas produções acadêmicas, visto que os autores apresentaram a conceituação genérica do modelo TPACK. Apesar da relevância dos criadores do modelo TPACK, faz-se importante relatar os autores contemporâneos e as pesquisas científicas mais recentes.

Cabe salientar a precariedade em estudos empíricos, sendo que, dentre os encontrados, a maioria são pesquisas teóricas e revisões da literatura. Com intuito de proporcionar avanços na utilização do modelo TPACK, destaca-se a importância de estudos exploratórios e investigativos, de forma empírica, com a coleta de dados diretamente com todos aqueles envolvidos no processo de ensino e aprendizagem

Observamos aspectos relevantes a serem considerados em futuras pesquisas, os quais destacamos: (1) limitação da infraestrutura escolar e dos alunos, são fatores que influenciam a utilização de tecnologias digitais no processo de ensino-aprendizagem; (2) faltam estudos voltados para a Formação Inicial e Educação Profissional Técnica, uma vez que as pesquisas apresentam a utilização do modelo teórico para Formação Continuada dos Professores na Educação Básica.

Vale ressaltar que alguns estudos trazem fundamentos descritos na Base Nacional Comum Curricular (BNCC) para justificar a importância do TPACK para a formação docente, visto que o modelo integra a tecnologia no ensino pedagógico de conteúdo, no entanto, há necessidade de aprofundamento nas pesquisas.

Concluímos que a tecnologia digital é apresentada como um conhecimento essencial para a atualidade, visto que dialoga com a possível realidade dos alunos e com as demandas sociais, potencializando o seu aprendizado. No que tange à formação de professores, 
compreende-se a relevância do modelo TPACK de forma que proporcione saberes docentes essenciais para a atuação docente na era digital.

Nesse sentido, torna-se relevante futuras pesquisas que possibilitem a compreensão e importância da tecnologia dentro dos processos de ensino e aprendizagem, desde a formação inicial até em seus futuros processos formativos.

\section{Referências}

ANDRADE, Mariel José Pimentel de; ALENCAR, Anderson Fernandes de; COUTINHO, Clara Pereira. O TPACK e a taxonomia dos tipos de atividades de aprendizagem: frameworks para integração da tecnologia na educação. Educação e Cultura Contemporânea, [S.L.], v. 16, n. 43, p. 169-189, 2019. GN1 Genesis Network. http://dx.doi.org/10.5935/2238-1279.20190009. Disponível em: http://periodicos.estacio.br/index.php/reeduc/article/viewArticle/5907. Acesso em: 12 set. 2020.

ARAÚJO, Kátia Soane Santos; HETKOWSKI, Tânia Maria. Educação científica e a teoria do conhecimento tecnológico e pedagógico do conteúdo (TPACK) na educação. In: seminário internacional de representações sociais, subjetividade e educação, 4., 2017, Curitiba. Anais [...]. Curitiba: Educere, 2017. p. 5940-5954. Disponível em: https://educere.bruc.com.br/arquivo/pdf2017/24461_12524.pdf. Acesso em: 12 set. 2020.

CIBOTTO, Rosefran Adriano Gonçales; OLIVEIRA, Rosa Maria Moraes Anunciato. TPACK - Conhecimento tecnológico e pedagógico do conteúdo: uma revisão teórica. Revista

Imagem da Educação, Maringá, v. 7, n. 2, p. 11-23, jan. 2017. Disponível em: http://periodicos.uem.br/ojs/index.php/ImagensEduc/article/view/34615. Acesso em: 12 set. 2020.

ESPÍNDOLA, Marina Bazzo de; GIANNELLA, Taís Rabetti. Integração de Tecnologias Digitais de Informação e Comunicação no Ensino de Ciências: Contribuições Do Modelo Do Conhecimento Pedagógico-Tecnológico Do Conteúdo. Revista Educere Et Educere, Paraná, v. 14, p. 1-26, jul. 2019. Disponível em:

http://saber.unioeste.br/index.php/educereeteducare/article/view/18287. Acesso em: 12 set. 2020.

ESPÍNDOLA, Marina Bazzo de; RESES, Gabriela de Nóbrega; RAMOS, Vinicius Faria Culmant. Formação docente para o ensino superior mediado por Tecnologias Digitais de Informação e Comunicação: uma proposta baseada no modelo do Conhecimento Pedagógico Tecnológico do Conteúdo. Research, Society And Development, São Paulo, v. 9, n. 7, p. 1-19, jan. 2020. Disponível em: https://rsdjournal.org/index.php/rsd/article/view/4694. Acesso em: 12 set. 2020.

ESPÍRITO SANTO, Eniel do; CARDOSO, Ariston De Lima; SANTOS, Adilson Gomes dos. Conhecimento Tecnológico Pedagógico do Conteúdo (TPACK). CIET: EnPED, 2018. Disponível em: https://cietenped.ufscar.br/submissao/index.php/2018/article/view/264. Acesso em: 12 set. 2020. 
GIL, Henrique. A passagem da Web 1.0 para a Web 2.0 e... Web 3.0: potenciais consequências para uma «humanização» em contexto educativo. Educatic: Boletim Informativo, p. 1-2, 2014.

LANG, Affonso Manoel Righi. O desenvolvimento do conhecimento pedagógico tecnológico do conteúdo de professores do ensino fundamental. 2016. 132 f. Dissertação (Mestrado) - Curso de Desenvolvimento Humano e Tecnologias, Instituto de Biociências, Universidade Estadual Paulista, Rio Claro, 2016. Disponível em:

https://repositorio.unesp.br/handle/11449/141532. Acesso em: 12 set. 2020.

LEITE, Bruno Silva. Revisão sistemática sobre as produções científicas da Revista Tecnologias na Educação. Revista Tecnologias na Educação, Minas Gerais, v. 25, n. 10, p. 1-16, jul. 2018.

MANEIRA, Simone; GOMES, Maria João. Professores e TPACK: uma revisão sistemática da literatura. VII Congresso Mundial de Estilos de Aprendizagem: livro de Atas, Portugal, ano 1, v. 1, p. 1345-1360, 2016.

MELO, Elvis Medeiros de. A integração do smartphone em sequência de ensino de estatística para o desenvolvimento do conhecimento tecnológico, pedagógico e do conteúdo. 2019. 212 f. Dissertação (Mestrado) - Curso de Programa de Pós-Graduação em Inovação em Tecnologias Educacionais, Instituto Metrópole Digital, Universidade Federal do Rio Grande do Norte, Natal, 2019. Disponível em:

https://repositorio.ufrn.br/jspui/handle/123456789/28590. Acesso em: 12 set. 2020.

MISHRA, Punya; KOEHLER, Matthew J. Technological pedagogical content knowledge: A framework for teacher knowledge. Teachers college record, v. 108, n. 6, p. 1017-1054, 2006. Disponível em: https://www.learntechlib.org/p/99246/?nl=1. Acesso em: 12 set. 2020.

MORAN, J.M. Novas Tecnologias e Mediação Pedagógica. Campinas: Papirus, 2001.

NOGUEIRA, Fernanda; PESSOA, Teresa; GALLEGO, Maria-Jesus. Desafios e oportunidades do uso da tecnologia para a formação contínua de professores: uma revisão em torno do TPACK em Portugal, Brasil e Espanha. \#Tear: Revista de Educação, Ciência e Tecnologia, v. 4, n. 2, p. 1-20, 2015.

OLIVEIRA, Maíra Marques de. Conhecimento pedagógico e tecnológico do conteúdo na formação de professores na educação científica e tecnológica. 2017. 164 f. Dissertação (Mestrado) - Curso de Programa de Pós-Graduação em Educação Científica e Tecnológica, Universidade Federal de Santa Catarina, Florianópolis, 2017. Disponível em: https://repositorio.ufsc.br/handle/123456789/186140. Acesso em: 12 set. 2020.

PINHEIRO, Izoldi Klein; BENVENUTTI, Dilva Bertoldi; FAVRETTO, Jacir. Ambiente de aprendizagem: conhecimento tecnológico pedagógico do conteúdo. Revista Eletrônica de Educação, São Carlos, v. 14, p. 1-16, jan. 2020. Disponível em:

http://www.reveduc.ufscar.br/index.php/reveduc/article/view/3765. Acesso em: 12 set. 2020. 
ROCHA, Marcelo Augusto. O Conhecimento Tecnológico e Pedagógico do Conteúdo (TPACK) aplicado ao ensino de Geografia. 2015. $255 \mathrm{f}$. Tese (Doutorado) - Curso de Programa de Pós-graduação em Ensino de Ciências e Educação Matemática, Universidade Estadual de Londrina, Londrina, 2015.

ROCHA, Marcelo Augusto; SALVI, Rosana Figueiredo. O conhecimento tecnológico pedagógico do conteúdo como aporte para o emprego das tecnologias digitais de informação e comunicação no ensino de Geografia. Giramundo, Rio de Janeiro, v. 8, n. 5, p. 57-68, jun. 2016. Semestral. Disponível em:

https://dspace.unila.edu.br/handle/123456789/5240. Acesso em: 12 set. 2020

ROLANDO, Luiz Gustavo Ribeiro. Um exame da percepção de professores de Biologia acerca de suas bases de conhecimento tecnológico pedagógico do conteúdo. $2017.162 \mathrm{f}$. Tese (Doutorado) - Curso de Pós-Graduação em Ensino em Biociências e Saúde, Instituto Oswaldo Cruz, Rio de Janeiro, 2017.

ROLANDO, Luiz Gustavo Ribeiro; LUZ, Maurício Roberto Motta Pinto da; SALVADOR, Daniel Fábio. O Conhecimento Tecnológico Pedagógico do Conteúdo no Contexto Lusófono: uma revisão sistemática da literatura. Revista Brasileira de Informática na Educação, Porto Alegre, v. 23, n. 3, p. 174-190, 2015. Disponível em:

https://br-ie.org/pub/index.php/rbie/article/view/3331. Acesso em: 12 set. 2020.

SANTOS NETO, Raul dos; SILVA, Leonardo Alves; DUQUE, Lucas; STRUCHINER, Miriam. Conhecimento Tecnológico Pedagógico do Conteúdo e a Formação de Professores de Ciências: uma revisão sistemática. In: XI ENCONTRO NACIONAL DE PESQUISA EM EDUCAÇÃO EM CIÊNCIAS - IX ENPEC, 6., 2017, Santa Catarina. Anais [...]. Santa Catarina, 2017. v. 1, p. 1-8. Disponível em:

http://www.abrapecnet.org.br/enpec/xi-enpec/anais/resumos/R1513-1.pdf. Acesso em: 19 set. 2020.

SANTOS, Luciane Mulazani dos; EGIDO, Sidnéia Valero. Conhecimento Pedagógico e Tecnológico do Conteúdo. In: Colóquio Luso-Brasileiro de Educação, 2., 2016, Joinville. Anais [...]. Joinville: Udesc, 2016. p. 704-704. Disponível em:

https://periodicos.udesc.br/index.php/colbeduca/article/view/8330. Acesso em: 19 set.

2020.SANTOS, Matheus Henrique Silva de Lima. Uma Análise de um Livro Didático de Matemática à Luz do Conhecimento Tecnológico Pedagógico do Conteúdo (TPACK). 2019. 52 f. TCC (Graduação) - Curso de Licenciatura em Matemática, Departamento de Matemática, Universidade Federal Rural de Pernambuco, Recife, 2019.

SHULMAN, Lee. Those Who Understand: Knowledge Growth in Teaching. Educational Researcher, v. 15, n. 2, p. 4-14, 1986.

SOUSA, Sidinei Oliveira; TERÇARIOL, Adriana Aparecida Lima; GITAHY, Raquel Rosan Christino. Conhecimento Tecnológico Pedagógico do Conteúdo: construção de conceitos e habilidades didáticas. Perspectiva, v. 35, n. 4, p. 1215-1239, 2017. 
TRIDICO, Diego Henrique de Moraes. Contribuições de um Curso de Formação Continuada para Professores dos Anos Iniciais no Desenvolvimento do Conhecimento Tecnológico, Pedagógico e de Conteúdo Algébrico. 2019. 129 f. Dissertação (Mestrado) Curso de Mestrado Profissional em Educação Escolar, Faculdade de Educação, Universidade Estadual de Campinas, Campinas, 2019. Disponível em:

http://repositorio.unicamp.br/handle/REPOSIP/343854. Acesso em: 29 set. 2020.

WENTZ, Fabiane Malakowski de Almeida. O uso do conhecimento tecnológico, pedagógico e de conteúdo integrado a taxonomia digital de Bloom para o ensino de química. 2018. 168 f. Dissertação (Mestrado) - Curso de Programa de Pós-Graduação em Educação em Ciências: Química da Vida e Saúde, Centro de Ciências Naturais e Exatas, Universidade Federal de Santa Maria, Santa Maria, 2018. Disponível em https://repositorio.ufsm.br/handle/1/14775. Acesso em: 30 set. 2020.

Recebido em dezembro de 2020.

Aprovado em maio de 2021. 